\title{
Die Performanz des Schmerzes. Poetische Inszenierungsstrategien von passio und compassio bei Oswald von Wolkenstein
}

\section{Fragestellung, Gegenstand, Kontext}

Der folgende Beitrag widmet sich der poetischen Inszenierung von passio und compassio sowie den textuellen Strategien der Rezeptionslenkung in Oswalds von Wolkenstein fünfstrophigem Marienlied Hört zu (Kl. 114). ${ }^{1}$ Während die übrigen Marienlieder $^{2}$ unter verschiedenen Perspektiven, beispielsweise dem Spannungsfeld von Innovation und Tradition, ${ }^{3}$ in der Forschung berücksichtigt wurden, fand Kl. 114 bisher nur am Rande Beachtung. ${ }^{4}$ Unter dem Aspekt der poetischen Erneuerung scheint das besagte Marienlied in der Tat wenig ergiebig. Das Stück, das die compassio Mariae reziprok zum Passionsgeschehen inszeniert, bewegt sich mit der auf den Affekt der Rezipienten und Rezipientinnen zielenden Narrativierung des Leidenswegs Jesu und der Trauer der Gottesmutter Maria im gängigen Rahmen spätmittelalterlicher Passionsfrömmigkeit. Bemerkenswert an besagtem Lied sind allerdings die poetischen Strategien der compassio-Inszenierung, die mit Hilfe einer komplexen, textintern die Sprecherinstanz und die Figur Marias sowie textextern die Hörerschaft einschließenden Kommunikationssituation eine meditative Versenkung in das vergangene Heilsgeschehen an-

1 Die Liedzitate des Beitrags folgen der Ausgabe Burghart Wachingers, Die Lieder Oswalds von Wolkenstein.

2 Diese machen in etwa zehn Prozent des Euvres Oswalds aus. Vgl. Hartmann: Madonnenbilder, S. $72-73$.

3 So betrachtet etwa Sieglinde Hartmann: Oswald, S. 364, das Marienlied Es leucht durch grau (Kl.34) als Ausdruck einer „neue[n] Form intim-persönlicher Marienfrömmigkeit“, für die Oswald, „ähnlich wie in der Liebeslyrik, eine neue Sprache beseelter Sinnlichkeit entwickelt.“ Freimut Löser, der die Marienlieder typologisiert, interessiert vornehmlich die zum Teil greifbare Überschneidung von weltlicher und geistlicher Motivik, welche die Figur Marias sowie die Beziehung des sich mitunter als ,Wolkensteiner identifizierenden Text-Ichs zu Gott prägt. Vgl. Löser.

4 Hervorzuheben sind an dieser Stelle die Beiträge Franz Viktor Spechtlers und Stefan Rosmers. In seiner Untersuchung der Marienlieder Oswalds charakterisiert Spechtler, S. 189, Kl. 114 als szenisches Lied, das aufgrund der Passionsthematik liturgisch dem Osterfestkreis zuzuordnen sei. Rosmer, S. 259f. geht unter dem Aspekt der Korrelation von metrisch-melodischer Form sowie Inhalt und sprachlicher Gestalt bei den Marienliedern Oswalds auch auf Kl. 114 ein, das er in die Tradition der Sangspruchdichtung und meisterlichen Liedkunst stellt.

Ә OpenAccess. (C) 2020 Eva Rothenberger, publiziert von De Gruyter. (cc) BY Dieses Werk ist lizenziert unter der Creative Commons Attribution 4.0 International. https://doi.org/10.1515/9783110666816-008 
streben. Durch die sinnliche Vergegenwärtigung des Leids auf Figurenebene sowie durch direkte Ansprachen an das Publikum des Liedes wird dieses zum performativen Nachvollzug der compassio Marias und damit letztlich der passio Jesu aufgerufen. Die theologische Reziprozität von passio und compassio schlägt sich in Kl. 114 nicht nur auf der semantischen Ebene nieder, sondern wird auf der formalen Ebene fortgesetzt, sodass sich das Marienlied als vielschichtige Komposition erweist.

Das performative Textpotential grenzt das besagte Marienlied von den beiden Weihnachtsliedern Oswalds, In Suria ein braiten hal (Kl. 35) und Freu dich, durchläuchtig junkfrau zart (Kl.126), sowie seinem zweiten Passionslied Der himel fürst uns heut bewar (Kl. 29) ab. ${ }^{5}$ Die genannten Stücke thematisieren zwar ebenfalls die soteriologische Wirkung des Erlösungstodes Jesu, verzichten allerdings auf eine performative, auf die emotionale Teilhabe der Rezipienten und Rezipientinnen zielende Inszenierung des Passionsgeschehens.

Aufgrund der intendierten Textwirkung, der Imagination der Passion Christi vor dem inneren Auge der Zuhörerschaft, steht das Marienlied Hört zu im thematischen Umfeld der volkssprachlichen Marienklagen, die seit dem dreizehnten Jahrhundert ${ }^{6}$ sowohl als selbstständige Stücke als auch in die spätmittelalterlichen Passionsspiele eingebunden überliefert sind. ${ }^{7}$ Im Zentrum der Marienklage steht bekanntermaßen die Figur der mater dolorosa, die im Kontext der Karfreitagsliturgie mit ihrer compassio zur Identifikations- und Stellvertreterfigur für die anwesende Gemeinde avanciert: ${ }^{8}$ „Das physische Leiden Christi am Kreuze wird hier gleichsam in seiner Umformung als seelisches Leid im Herzen der Mutter

5 Kl. 29 und Kl. 114 verweisen intertextuell mit Hilfe eines semantisch äquivalenten Zitates aufeinander: kreutzlicher hank, erlös uns ewikleichen (Kl. 29: II,9) steht [...] helf uns sein chreutzlich hankh (Kl. 114: V,19) gegenüber.

6 Vgl. Lipphardt, S. 198. Als die wesentlichen lateinischen Vorbilder der deutschsprachigen Marienklagen gelten die Sequenz Planctus ante nescia (vor Mitte des zwölften Jahrhunderts; AH, Bd. 20, Nr. 199, S. 156 -158) sowie der Tractatus beati Bernhardi de planctu beatae Mariae virginis (nach 1205) und die Interrogatio Sancti Anselmi de Passione (nach 1238). Vgl. Satzinger, Ziegeler, S. 244f. Die genannten Texte geben den deutschsprachigen Marienklagen im Wesentlichen die „Form (,monologisch‘, ,dramatisch‘, erzählend), Struktur (,unmittelbare‘ oder ,nachträgliche‘ Vergegenwärtigung der Passion) und theologischen Sinn vor [...].“ Ebd., S. 245.

7 Vgl. Mehler, Bd. 1, S. 17. Auf die Gattungsdiskussion rund um die Marienklage hinsichtlich der Kategorie des Dramatischen (vgl. hierzu Petersen, der den Terminus des Rituals vorzieht) sowie auf eine Differenzierung verschiedener Klagetypen (vgl. hierzu Mehler, Bd. 1, bes. S. 25-31; 186195) kann an dieser Stelle nicht eingegangen werden.

8 Siehe Lipphardt, S. 199. Auch die selbstständigen Marienklagen stehen den Passionsspielen insofern als ,dramatischer Texttyp nahe, als dass sie beispielsweise in Spielhandschriften samt Regieanweisungen - teils mit beigegebenen Noten - aufgezeichnet sind und in einigen Handschriften sogar als ludus ausgewiesen werden. Vgl. Mehler, Bd. 1, S. 17. 
geschaut und miterlebt. “9 Diese durch die Klage evozierte Wirkung auf das Publikum wird, wie im Folgenden zu zeigen ist, in ähnlicher Weise auch in Oswalds Marienlied erzielt, das die Hörerschaft im Vollzug des Liedvortrags in das Geschehen um passio und compassio involviert. Die Figur Marias wird hierbei zur Mittlerin ihrer compassio, der Eintritt in die „Liebes- und Leidensgemeinschaft“ von Mutter und Sohn zeitigt soteriologische Wirkung. ${ }^{10}$ Ausgehend von der in der ersten Hälfte des fünften Jahrhunderts konziliarisch festgesetzten zweifachen Wesenheit Jesu als Mensch und Gott ${ }^{11}$ tritt in der monastischen Passionstheologie $^{12}$ des hohen Mittelalters zunehmend Christus als Mensch der Passion in den Vordergrund und steht im späten Mittelalter schließlich im Zentrum von Heilsgeschehen und Heilsbetrachtung. ${ }^{13}$ Wegweisend sind in dieser Entwicklungslinie unter anderem die Satisfaktionslehre ${ }^{14}$ des Anselm von Canterbury († 1109), welche die theologische Begründung für die Notwendigkeit der Menschwerdung Jesu liefert, sowie die Passionstheologie Bernhards von Clairvaux († 1153), in der die Passion Christi in einer bislang ungekannten Intensität im Mittelpunkt steht. ${ }^{15}$ Die Selbstentäußerung Gottes in der Inkarnation - die Menschwerdung und die Akzeptanz des Leidens gelten Bernhard als „die höchsten Offenbarungen der Liebe Gottes zu den Menschen“16 - bildet die Grundlage für die Erfahrbarkeit des Transzendenten in der Immanenz. Sie eröffnet einen affektiven Kontaktbereich zwischen Mensch und Gott, indem das durch den Gottessohn erfahrene

9 Lipphardt, S. 199.

$10 \mathrm{Kraß,S} .73$.

11 Der auf dem Konzil von Ephesus (431 n.Chr.) festgesetzte marianische Titel der Gottesgebärerin diente letztlich zur Sicherung des christologischen Dogmas der zweifachen Wesenheit Jesu als Gott und Mensch: Maria ist Mutter des Mensch gewordenen Gottessohnes, ohne jedoch selbst göttlich zu sein. Vgl. Beinert, S. 265. Die theologische Auseinandersetzung um die göttliche Wesenheit Jesu wurde schließlich auf dem im Jahr 451 abgehaltenen Konzil von Chalcedon mit der Aussage, Jesus sei eine Person in zwei Naturen, entschieden. Vgl. Berger, S. 124.

12 Der monastischen Theologie (u.a. vertreten durch Bernhard von Clairvaux), welche die menschliche ratio als unzulässiges Instrument des Glaubens wertet, steht die dialektische Theologie (u.a. vertreten durch Abaelard), die auf rationale Erklärung theologischer Mysterien und terminologische Präzision setzt, gegenüber. Vgl. Langer, S. $46 \mathrm{f}$.

13 Vgl. die Darstellung der theologischen Positionen der Passionsfrömmigkeit und ihrer chronologischen Entwicklung von der Antike bis zum späten Mittelalter bei Berger, S. 123-155.

14 Anselm von Canterbury beeinflusst in seinem Werk Cur Deus homo maßgeblich die Satisfaktionslehre, die sich mit der Frage nach der Notwendigkeit des Leidens Gottes auseinandersetzt. Demnach könne die Wiederherstellung der Weltordnung nach dem Sündenfall lediglich durch die Genugtuung Gottes in Form der freiwilligen Menschwerdung des Gottessohnes und dessen Akzeptanz des Todes am Kreuz wiederhergestellt werden. Vgl. Ruh, S. 33 u. 35.

15 Vgl. Berger, S. 126 u. 131.

16 Langer, S. 59. 
menschliche Leid „den Menschen in die Lage [versetzt], mit Gottes Leiden aus Mitleid selbst Mitleid zu haben [...]. “17 Das Leid Jesu, das in und über die compassio Mariae fassbar wird, erweist sich hierbei als entscheidendes Movens, denn die Hervorhebung des persönlichen Erlebens und die Versinnlichung des Transzendenten führen gemäß Ursula Schulze dazu, dass „Gott nicht mehr nur erhöht und abgerückt im Gegensatz gedacht wird, sondern im affektiven Nachvollzug markanter Phasen des Erdenlebens Christi erfassbar erscheint. “18 Maria kommt in diesem Zuge eine entscheidende Mittlerfunktion zwischen Immanenz und Transzendenz zu - in ihrer Rolle als mitleidende Muttergottes avanciert sie zur Identifikationsfigur für die Menschen und erhält bezüglich ihrer emotionalen Teilhabe am Leid Jesu Vorbildfunktion. Die compassio steht im Zentrum der imitatio Christi - mit Hilfe der lesenden oder hörenden Rezeption der Passionsgeschichte soll der Mensch über das Mittel der Betrachtung ${ }^{19}$ der Leidensstationen Jesu (meditatio, contemplatio) zum Mitleiden (compassio) bis hin zur Nachfolge (imitatio) gelangen. ${ }^{20}$ Während der Wandel vom deus gloriae zum homo crucifixus mit Bernhard zwar vollzogen ist, jedoch die göttliche Wesenheit Jesu nach wie vor gleichberechtigt neben der menschlichen steht, ${ }^{21}$ setzt die Passionsfrömmigkeit des Spätmittelalters schließlich einseitig auf die sinnliche Vorstellung des Leidens Jesu und damit auf dessen Menschwerdung. ${ }^{22}$ Diese Fokussierung auf den leidenden, irdischen Christus schlägt sich in den spätmittelalterlichen Passionstraktaten, welche Predigten, epische Texte sowie Passionsspiele theologisch fundieren, ${ }^{23}$ in einer zunehmend drastischen Ausschmückung von Qual und Folter nieder, ${ }^{24}$ im Falle der Passionsspiele in einer deutlichen Ausweitung der

17 Ebd. Das Ineinandergreifen von Leid und Liebe als Voraussetzung für die menschliche Erfahrbarkeit Gottes im Diesseits legt Bernhard im 43. Sermo seiner Hoheliedauslegung theologisch dar. Vgl. Langer, S. 60.

18 Schulze: Schmerz, S. 211.

$19 \mathrm{Zu}$ diesem Zweck berichten die spätmittelalterlichen Passionstraktate die Passionsgeschichte auf der Grundlage etwa der apokryphen Erzähltradition (vgl. das Evangelium Nicodemi) zum Teil detaillierter und anschaulicher als die Evangelien selbst. Vgl. Ruh, S. 20.

20 Siehe ebd. In der scholastischen Seelenanthropologie und Erkenntnislehre wird die Wirkmacht der inneren Bilder des Heilsgeschehens, welche die memoria prägen und so auf den inneren Zustand des Menschen einwirken, hervorgehoben. Die Nachfolge Christi konstituiert sich hierbei mit Hilfe der ,Überbildung' des betrachtenden Menschen durch das Bild der Vollkommenheit, d.h. Christus. Vgl. Lentes, v. a. S. 182f.; 186-190.

21 So ist gemäß Bernhard „die Betrachtung der äußeren Leiden Christi nur die eine Seite der Erkenntnis Jesu, sie muß weiterleiten zur Gottheit Christi.“ Berger, S. 132f.

22 Vgl. ebd., S. 129 u. 133.

23 Vgl. Ruh, S. 19.

24 Vgl. Berger, S. 148f. 
Gewaltszenen. ${ }^{25}$ In diese auf der spätmittelalterlichen Passionsfrömmigkeit fußenden Erzähltradition, welche die Schmerzensmetaphorik intensiviert, reiht sich auch Oswalds Marienlied ein, das im Folgenden hinsichtlich der auf Teilhabe am Heilsgeschehen zielenden textinternen Signale analysiert werden soll.

Im Unterschied zur performativen Emotionsforschung, die unter ,Performanz den Prozess versteht, „durch den sich ein kulturelles Ereignis - wie die Aufführung oder Lektüre eines literarischen Werkes - situational und je einmalig voll-

zieht [...]“, ${ }^{26}$ geht es mir weniger um die Frage nach einem empirischen Liedvortrag. Im Vordergrund der folgenden Ausführungen steht vielmehr die vor dem inneren Auge des Rezipienten und der Rezipientin imaginierte Vergegenwärtigung des Passionsgeschehens und die evozierte Teilhabe an der compassio der Gottesmutter. Der Performanzbegriff umfasst hierbei die auf die textinterne Rezeptionslenkung hinweisenden performativen Textsignale. ${ }^{27}$

\section{Performative Textsignale in Kl. 114}

Bereits die Anfangsworte des Marienliedes markieren das performative Potential des Textes. Die Apostrophe Hört zu eröffnet eine Kommunikationssituation zwischen der Sprecherinstanz des Liedes und dessen Rezipientinnen und Rezipienten, die dazu aufgefordert werden, ihre Aufmerksamkeit auf eine ellentleich[e] mär (I,1) zu richten. Diese erweist sich im Folgenden als die an Maria überbrachte Kunde von der Gefangennahme ihres Sohnes (I,2-3). Die näheren Umstände werden erst zu Beginn des zweiten Stollens der Strophe genannt: Ein geflohener Jünger, ein Augenzeuge der Verhaftung, verkundt der frauen, als man redt, / wie man den fuerte groblich roch, ${ }^{28}$ / den si lieplich erzogen hett (I,8-11). Nach der Benachrichtigung Marias über die Gefangennahme Jesu setzt der Bericht über das eigentliche Passionsgeschehen mit seinen diversen Wegstationen ein: beginnend

25 Vgl. Egidi, S. 181.

26 Eming u.a., S. 217.

27 Der Begriff des Performativen umfasst ebenso wie in der performativen Emotionsforschung „Handlungen oder Interaktionen [...], die als Vollzug, Vergegenwärtigung oder Erzeugung charakterisiert werden können.“ Eming u.a., S. 217.

Bezüglich der Passionsspiele verzichten Ursula Schulze und Margreth Egidi auf die terminologische Differenzierung von Performanz und Performativität. So dient Schulze: Schmerz, S. 216, Anm. 16, der Performativitätsbegriff sowohl für die Aufführung als auch die Textsignale, die eine solche konditionieren. Margreth Egidi unterscheidet wiederum zwei Darstellungsmodi der Performativität, „einerseits die Performativität der theatralen Mimesis, andererseits eine ,innere Performativität‘ im Sinne des Mitvollzugs der Passion durch den Betrachter.“Egidi, S. 182. 28 roch von rou, rouch Adj. ,roh‘ (Le II, Sp. 510), im Sinne von ,grob‘. 
mit der Verhaftung Jesu (I,12-14), gefolgt von der Anklage vor dem Richter (II,5-7), der Verhaftung (II,12-14) und der Verleugnung durch Petrus (II,17) bis zur Geißelung (III,8-14), der Dornenkrönung (IV,1-4) und der Kreuzauferlegung (IV,5-10); abschließend folgen die Kreuzigung (V,8-11) sowie die Grablege (V,12-13). Während sich die Schmerzens- und Leidensmetaphorik in der ersten Liedstrophe zunächst auf wenige Ausdrücke beschränkt, wie beispielsweise groblich roch (I,10), mit grosser smach (I,11), ellend[e] gach (I,11) sowie miche[l] praus (I,13), nehmen die Schmerzensmetaphern im Abgesang, der sich auf die compassio der Gottesmutter konzentriert, zu: O frau, wie bitter was der smerz, / den da empfieng dein keuschlich herz, / als es erhort das senlich mort (I,15-17). Zunächst metaphorisch im Motiv des Leidensschwertes gefasst, wird die Reaktion Marias auf die schlechte Nachricht in den letzten Versen der Strophe zur unmittelbaren körperlichen Reaktion gesteigert: Der Schmerz der Gottesmutter wirkt sich auf leib, varb und gestalt [...] aus (I,18); die erste von insgesamt drei Ohnmachten Marias innerhalb des Liedes ist die Folge (I,18-19).

Mit Hilfe der Überschneidung verschiedener Verkündigungsszenen in der Eingangsstrophe wird eine Kommunikationsgemeinschaft inszeniert, welche die textimmanente Ebene (Maria, Sprecherinstanz) und die textexterne Ebene (Publikum) des Liedes gleichermaßen einschließt, und sowohl die Figur Marias als auch das Publikum des Liedes zu Zeugen des Geschehens erhebt. Textintern deutet sich die Verkündigung der jungfräulichen Geburt an Maria durch den Erzengel Gabriel im Zitat des englischen Grußes an (I,7: ,ave an mail empfieng [...]); es folgt der Zeugenbericht des Jüngers über die Verhaftung Jesu (I,8-10); die Sprecherinstanz wiederum unterrichtet das textexterne Publikum, das auf diese Weise zu Zeugen des narrativierten Geschehens wird, von dem vergangenen Ereignis. Diese Überlagerung der unterschiedlichen Kommunikationsebenen ermöglicht eine imaginäre Interaktion zwischen den textintern und -extern am Geschehen Beteiligten: Jene an Maria übermittelte ellentleich $[e]$ mär, welche die ersten Liedverse als vergangenes Faktum anführen, wird im performativen Vollzug des Liedes für das zur Aufmerksamkeit aufgeforderte Publikum (V. 1: Hört zu [...]) nochmals vergegenwärtigt. Die so imaginierte Gemeinschaft ebnet in der ersten Liedstrophe den Weg zur Identifikation der Zuhörer mit der compassio der im Mittelpunkt des Liedes stehenden mater dolorosa, die sich im weiteren Textverlauf steigert. Der Schmerz wird zur performativen Emotion, die sich zunächst textintern auf der Figurenebene von Christus über Maria und schließlich auf die Sprecherinstanz des Liedes überträgt - deren klagende Interjektion $O$ frau $[. .].(I, 15)$ steht in direkter Analogie zum Schmerz der Gottesmutter, der für das textexterne Publikum vergegenwärtigt wird.

In die zu Beginn der zweiten Strophe fortgeführte Apostrophe an Maria, welche das Motiv der compassio fortsetzt, schaltet sich die Sprecherinstanz des 
Liedes auf zweifache Weise wertend in das berichtete Geschehen ein: Zum einen beruhe die Anklage Jesu [...] vor des richters stab (II,7) auf falschen Vorzeichen (II,6), zum anderen seien die [u]nzälich klag und senlich mat (II,1) der Gottesmutter bedächtikleich und erlaubt (II,2). Die vorgebrachte Plausibilität und Nachvollziehbarkeit des menschlichen Schmerzes der ihres Kindes beraubten Mutter (II,4) mündet in eine weitere direkte Anrede an die Gottesmutter, in der sich die Sprecherinstanz erstmals als Text-Ich zu Wort meldet: $O$ junkfröuleiche raine maid, / mich wundert, das dir nit erbrach / dein keuschlich herz von grossem laid, / als du den herren hort und sach (II,8-11). Die Schwere des Leids und die compassio Marias werden an dieser Stelle vor dem Hintergrund der scholastischen Satisfaktionslehre ${ }^{29}$ mit der herausgehobenen Stellung als göttlich erwählte Gottesmutter und ihrer tugendhaften Vorbildhaftigkeit ${ }^{30}$ kontrastiert und auf diese Weise intensiviert.

Die dritte Strophe, die sich erneut an Maria wendet, beginnt mit einem Marienpreis, der die bekannten Topoi der mittelalterlichen Marienverehrung aufruft (III,1-4: göttliche Erwählung Marias und Erhabenheit über alle Frauen). Die so formulierte exponierte Stellung der Gottesmutter wird abermals der Schwere ihres zu ertragenden Leids gegenübergestellt und die Dimension des Schmerzes somit gesteigert, wie die Frage des Text-Ichs veranschaulicht: wie mocht dein adelich natur / erzeugen durch ain zarten leib / den grossen schrick und scharpfen blick / des gaisel slag, von dem du lag, / geswindlich auf die erden viel (III,3-7). Die Jesus treffenden und verwundenden Schläge in der Geißelungsszene (III,8-14) werden zum Analogon der zuvor angeführten Verwundung der Mutter Jesu: Der Maria zu Boden werfende gaisel slag metaphorisiert die zweite Ohnmacht der Gottesmutter im Angesicht des Leids ihres Sohnes. Ebenso wie am Ende der ersten Strophe, in der Marias Ohnmacht durch die Nachricht über die als Untat deklarierte Verhaftung ihres Sohnes (I,17) ausgelöst wird, basiert auch an dieser Stelle die affektive, den gesamten Körper Marias involvierende Reaktion auf der sinnlichen Wahrnehmung - nun ist sie allerdings selbst Augenzeugin des Geschehens (III,5). Bereits zuvor weist das Prädikat erzeugen (III,4) den Figurenkörper explizit als Spiegelbild des Leidens ihres Sohnes aus - die passio Christi evoziert die compassio Mariae, der Schmerz des Gottessohnes erfasst die Gottesmutter gleicher-

29 Die theologische Erörterung der Opposition von göttlicher Allmacht und einer soteriologischen Notwendigkeit von Leid und Tod des Gottessohnes war in den breit überlieferten volkssprachlichen Passionstraktaten des späten Mittelalters allgegenwärtig. Vgl. Ruh, S. 17 u. 38.

30 Bedenkt man die Deutung der Frage Marias auf die Verkündigung der Geburt Jesu, wie eine Empfängnis möglich sei, als votum virginitatis (d.h. als Akzeptanz der ihr zugedachten Rolle im göttlichen Heilsplan) durch Augustinus, schwingt als Konnotat der Jungfräulichkeit Marias stets ihre Vorbildhaftigkeit in Tugend und im Glauben mit. Vgl. Gössmann, S. 17 u. 32. 
maßen. Die Schmerzensmetaphorik, die sich bislang überwiegend auf die Figur Marias konzentrierte, verlagert sich im zweiten Stollen der Strophe auf Christus. Der durch die Geißelschläge rot verfärbte Körper des nackten, an eine Säule gebundenen Christus (III,8-14), der das Passionsgeschehen plastisch veranschaulicht, hebt die menschliche Wesenheit des Gottessohnes besonders hervor, die wiederum mit der menschlichen Eigenschaft der Mutterliebe im Abgesang der Strophe korrespondiert: Zart minikliche kaiserin, / wie was betrübt herz, muet und sinn, / do man verwundt des himels grund / spotleichen schaut! / o edle braut, / wie was dein lieber sun so krankh (III,15-19).

In der anschließenden Szene der Dornenkrönung in der vierten Strophe wird das Leid, das durch das tiefe Eindrücken der Dornenkrone seitens der Peiniger noch drastischer dargestellt wird und Jesus in seinem ganzen menschlichen Leid zeigt, erneut über das Motiv des Blutes veranschaulicht: Mit ainem kranz von dornen scharf / der himel fürste ward verkrönt, / tiefflich gedruckt, das sich entwarf / mit bluet sein antlutz, houbt betrönt (IV,1-4). Auch die anschließende Kreuzauferlegung im zweiten Stollen der Strophe offenbart Jesus in seiner menschlichen Schwäche, als er das Kreuz [...] von krankhait nicht enmocht / allain getragen von der erd, / wann es zu swärlich was geblocht (IV,8-10). Maria, die bisher als hörende (Strophe I) oder sehende (Strophe III) Zeugin und somit lediglich indirekt am Passionsgeschehen beteiligt war, schaltet sich beim Anblick des geschwächten Sohnes erstmals aktiv ein: und das ersach sein mueter werd, / in seim ellend was si behend, / von rechter gier im hilflich schier, / des man ir nicht verhengen wolt (IV,11-14). Die liebende Bindung zwischen Mutter und Sohn, die seit der dritten Strophe thematisiert wird und in dem Versuch der fürsorglichen Mutter, dem leidenden Sohn zu Hilfe zu kommen, kulminiert, wird innerhalb der Liednarration zum entscheidenden Anknüpfungspunkt für die Identifikation der Rezipientinnen und Rezipienten mit der compassio Mariae. Denn die in der Szene der Kreuztragung thematisierte Vereinigung von Mutter und Sohn in Liebe und Schmerz mündet im Abgesang in eine Apostrophe, die im Kontext der Inszenierung von compassio als Kulminationspunkt des gesamten Liedes bezeichnet werden kann. Die Verse appellieren an die Lebenswirklichkeit der Hörerschaft, maßgeblich der weiblichen: Bedenk ain jede mueter das, / wenn si ir kind in solcher mass / säch vor ir steen und darnach geen / zu seinem tod mit solcher not, / wie ser betrüebt wer ir gedankh (IV,15-19). Der etwa aus der Tradition der Passionsspiele bekannte Gestus der sich an die anwesenden Mütter wendenden Figur Marias ${ }^{31}$

31 Eine solche Hinwendung der Figur Marias an die Mütter findet sich beispielsweise in den Klageszenen des ,Egerer Passionsspiels‘ (um 1500), das freilich zeitlich nach Oswalds Wirken liegt. Dort verlässt Maria die Handlungsebene, als sie das Publikum zur compassio auffordert und 
schließt im übertragenen Sinn das gesamte Publikum mit ein. Da die Mutterfigur sowie mitunter auch die alltagsweltliche Szenerie der fürsorglichen Mutter Teil der persönlichen Erfahrung vieler Menschen ist, vermag die explizit an die weibliche Zuhörerschaft gerichtete Aufforderung zur Identifikation mit Maria als leidender Mutter geschlechterübergreifend zu wirken.

Die erneute Klage des Text-Ichs, welche die letzte Strophe einleitet, stellt das Lied schließlich in den größeren Kontext der compassio-Erzählungen: Awe, die ellend wainleich klag, / der man nicht vil geschriben vindt, / allain neur als die frau da pflag / umb ir keuschlich geboren kind (V,1-4). Welche Schrift(en) an dieser Stelle gemeint sind, wird nicht explizit erwähnt; bedenkt man allerdings die Tradition der lateinischen wie volkssprachlichen Marienklagen, in welchen ebenjene Trauer der Mutter um den Sohn im Zentrum steht, wird man vermutlich an die Bibel denken dürfen. Dort wird das durch die Folter und den Tod des Sohnes hervorgerufene Leid der Mutter lediglich an zwei Stellen thematisiert: bei der Schwertprophezeiung Simeons im Lukasevangelium (Lk 2,35) sowie im Motiv der am Fuß des Kreuzes stehenden Gottesmutter im Johannesevangelium (Joh 19,25). ${ }^{32}$ Vor diesem Hintergrund scheint sich Oswald mit Hilfe der Klage des TextIchs in seinem Marienlied implizit in die Erzähltradition der mittelalterlichen Marienklagen - als narrative Ergänzung zur Bibel - einreihen zu wollen.

Die vorletzten Stationen des Passionsgeschehens im zweiten Stollen (V,8-11: Kreuzigung; V,12-13: Grablege) münden in die abschließende Apostrophe an Maria, die sie das erste Mal persönlich bei ihrem Namen nennt und sie zugleich letztmalig in ihrer Rolle als mater dolorosa am Kreuz zeigt:

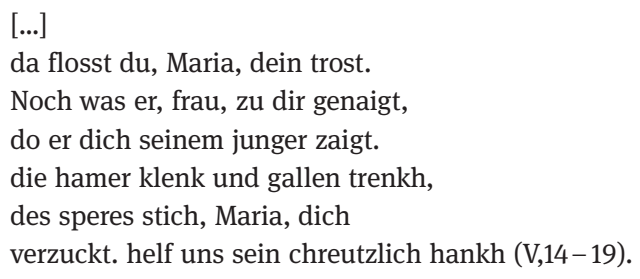

Erstmals spricht nun am Ende des Liedes, in Übereinstimmung mit dem alle Menschen einschließenden Erlösungstod Jesu, ein kollektives Wir. Das Text-Ich

speziell die Mütter anspricht: Alle, die do muetter sindt (EP 5942). Vgl. Schulze: Planctus, S. 202 (Zitat aus dem ,Egerer Passionsspiel‘ nach ebd.; Datierung des Spiels nach ebd., S. 199).

32 Das Markus- und Matthäusevangelium erwähnen lediglich, dass Maria gemeinsam mit anderen Frauen Jesus nachgefolgt sei und von Weitem Zeugin der Kreuzigung wurde (siehe Mk 15,40 f.; Mt 27,55f.). Vgl. Kraß, S. 93. 
erweist sich so in der Rückschau als Repräsentant des anwesenden Publikums. ${ }^{33}$ Die letzte Liedszene, in der sich der sterbende Jesus seiner Mutter zuneigt und sie seinem Lieblingsjünger anvertraut (V,15-16), ${ }^{34}$ mutet fast zärtlich an. Umso drastischer wirken die nochmals zu vernehmenden hamer klenk; der gallen trenkh $(V, 17)$ markiert schließlich den Zeitpunkt des Todes Jesu am Kreuz. Ähnlich wie zuvor beim Geißelschlag (Strophe III) wirkt auch hier des speres stich $(\mathrm{V}, 18)$ als jenes Maria selbst verwundende Leidensschwert - es folgt die dritte und letzte Ohnmacht der Gottesmutter: des speres stich, Maria, dich / verzuckt. helf uns sein chreutzlich hankh (V,18-19). Das Lied endet mit dem Hinweis auf die soteriologische Wirkung des Kreuzestodes Jesu, dem Ziel- und Endpunkt des im Lied narrativierten Passionsgeschehens.

\section{Formaler Einklang von passio und compassio}

Das Enjambement ${ }^{35}$ der beiden letztgenannten Schlussverse des Liedes kennzeichnet das Leid von Mutter und Sohn als formale Einheit. Die thematische und formale Korrespondenz von passio und compassio lässt sich auch im größeren Liedzusammenhang beobachten. Wie bereits in der Analyse anklang, stellen die einzelnen Strophenabschnitte jeweils eine weitestgehend in sich geschlossene semantische Einheit dar, d.h. die beiden Stollen sowie der Abgesang setzen jeweils thematisch neu ein ${ }^{36}$ und bilden metrisch-musikalisch eigenständige Blö-

33 An dieser Stelle wird die Analogie zum Typus der Marienklagen und zu einem ihrer Vorbilder, der Sequenz Planctus ante nescia (vgl. Anm. 6), deutlich, erweist sich in den Gattungen Hymnus und Sequenz ein Ich-Sprecher doch oftmals als Repräsentant des kollektiven Wir, d.h. der kirchlichen Gemeinde.

34 Die besagte Szene findet sich unter den vier Evangelien einzig bei Johannes (19,26f.).

35 Dieses ist auch in den beiden Handschriften B (Innsbruck, Universitäts- und Landesbibliothek, Cod. ohne Signatur) und c (Innsbruck, Landesmuseum Ferdinandeum, Cod. FB 1950) durch Versstriche eindeutig markiert.

36 Siehe ähnlich bereits Spechtler, S. 190f. Strophe I: Benachrichtigung der Rezipienten und Rezipientinnen (1. Stollen), Kunde von der Verhaftung Jesu an Maria (2. Stollen), Klage des Sprechers über das Leid Marias in Form einer Apostrophe (Abgesang); Strophe II: Fortsetzung der Apostrophe an Maria; Klage des Text-Ichs über die Anschuldigung Jesu (1. Stollen), Klage über die Passion Jesu (2. Stollen), Klage über die Verleugnung durch Petrus (Abgesang); Strophe III: Marienpreis und Klage über die Geißelung (1. Stollen), Geißelungsszene (2. Stollen), Apostrophe an Maria (Abgesang); Strophe IV: Dornenkrönung und Kreuzauferlegung (1. Stollen), Marias Versuch, dem Sohn zu Hilfe zu eilen (2. Stollen), Apostrophe an die Rezipientinnen (Abgesang); Strophe V: Klage des Text-Ichs über die mangelnde narratio der compassio Mariae (1. Stollen), Kreuzigung; im letzten Vers beginnt die Apostrophe an Maria (2. Stollen), die anschließend fortgesetzt wird (Abgesang). 
cke. ${ }^{37}$ Bis auf eine kleine Unregelmäßigkeit in der letzten Liedstrophe (Beginn der Apostrophe bereits im zweiten Stollen) erweisen sich die Strophenabgesänge jeweils als eine Apostrophe an Maria (Thema der compassio), Jesus (Thema der passio) oder die Rezipientinnen (Aufforderung zur compassio). Indem die Ansprache an Maria in der ersten Strophe mit derjenigen an Jesus in der zweiten Strophe parallelisiert wird, spiegeln sich compassio und passio auf der formalen Ebene: Die durch das Text-Ich vorgetragenen Klagen $O$ frau, wie bitter was der smerz $(\mathrm{I}, 15)$ und Ach got, wie ellend was der schein $(\mathrm{II}, 15)^{38}$ sind nicht nur formal äquivalent, indem sie jeweils den Abgesang der Strophe einleiten, sondern auch syntaktisch und morphologisch. Zudem rahmen die Ansprachen an Maria in den Abgesängen der ersten und fünften Strophe zum einen das Lied ein, zum anderen stehen die Anreden Marias zwischen den beiden Apostrophen an Christus und die Rezipientinnen. Somit erweist sich die Figur Marias als kompositorische Mitte des Liedes, das ${ }^{39}$ auf diese Weise einen bedeutenden Aspekt der mittelalterlichen Passionsfrömmigkeit formal abbildet, denn in ihrer Rolle als Gottesmutter steht sie als Vermittlerin der compassio zwischen den Polen der Immanenz und der Transzendenz. Selbst bei der letzten an Maria gerichteten Apostrophe, die bereits im letzten Vers des zweiten Stollens beginnt und damit von dem sonst üblichen Kompositionsschema abweicht, ließe sich überlegen, ob nicht auch an dieser Stelle der semantische Gehalt des Liedes mit dessen Form korrespondiert, diese formale Abweichung also intendiert ist. Denn ebenso wie die formale Gesetzmäßigkeit der Liedstrophen an dieser Stelle aufgebrochen ist, wird am Ende des Liedes die Leidens- und Liebesgemeinschaft von Gottesmutter und Gottessohn metaphorisch gesprochen durchlässig - am Fuß des Kreuzes kulminiert schließlich die gesamte Bewegungsinszenierung und Blickregie des Liedes, sodass sich der affektive Mitvollzug der compassio über den inneren Blick der Rezipienten und Rezipientinnen in der Heilstat des verstorbenen Christus bündelt.

Die Verschränkung von passio und compassio setzt sich selbst in der komplexen Reimanalogie der Liedstrophen fort. Während der erste und zweite Stollen jeweils im letzten Vers (V. 7 u. 14 jeder Strophe) vor allem auf die Lebens- und Passionsgeschichte Jesu Bezug nehmen, geht der jeweils letzte Vers einer Strophe

37 Vgl. Rosmer, S. 259. Diese Kompositionsstruktur steht nach ebd., S. 259f., in der Tradition der Sangspruchdichtung und Meisterliedtradition.

38 Spechtler, S. 191, bewertet die Interjektion Ach got hingegen nicht christologisch, sondern als Ansprache an Maria.

39 Der Apostrophe an Maria in Strophe I folgt die Apostrophe an Christus in Strophe II; in Strophe III wendet sich das Text-Ich erneut an Maria, bevor es sich in Strophe IV den Rezipientinnen zuwendet. Im Abgesang der Schlussstrophe wird nochmals Maria angerufen. 
(V. 19 jeder Strophe), der als Refrain alle Strophen miteinander verknüpft, auf die passio Jesu oder die compassio Marias ein:

Reimbezüge von erstem und zweitem Stollen:

I. Strophe: Geburt Jesu (gebar; V. 7) - Vorführen vor den Richter (der Juden schar; V. 14);

II. Strophe: Anklage Jesu (des richters stab; V. 7) - Prolepse auf Jesu Tod (grab; V. 14);

III. Strophe: Ohnmacht Marias (viel; V. 7) - Fesselung Jesu (riel; ${ }^{40}$ V. 14);

IV. Strophe: Kreuzauferlegung (sterben solt; V. 7) - Hinderung Marias, ihrem Sohn zu helfen (nicht verhengen wolt; V. 14);

V. Strophe: Erlösungstat Jesu (erlost; V. 7) - Maria als Trösterin (trost; V. 14).

Refrain:

I. Strophe: sankh (Maria fällt in Ohnmacht);

II. Strophe: schrankh (Jesus ist umringt von Feinden);

III. Strophe: krankh (Jesus ist geschwächt von der Folter);

IV. Strophe: gedankh (Apostrophe an die weibliche Zuhörerschaft des Liedes);

V. Strophe: hanckh (soteriologische Wirkung des Kreuzestodes).

Wie die Übersicht verdeutlicht, ergibt sich darüber hinaus eine heilsgeschichtliche Klammerung des Liedes, denn die Reimbezüge des jeweils ersten Stollenverses zitieren mit der Verkündigung der Geburt Jesu (gebar) und dem Hinweis auf die soteriologische Wirkung seines Todes (erlost) den theologischen Kerngedanken der Passionstheologie an. Der komplexe Aufbau des Liedes hebt sich ab von dessen metrischer Gleichmäßigkeit, die dem meditativen Charakter einer Passionsbetrachtung nahekommt. Bis auf zwei leicht abweichende Verse ${ }^{41}$ wird das Lied durch einen vierhebigen Jambus bestimmt, der jeweils mit einem unbetonten Auftakt beginnt und mit einer einsilbig vollen Kadenz schließt.

Die theologische Reziprozität von passio und compassio ist neben der semantischen und formalen Ebene außerdem überlieferungsgeschichtlich greifbar. Die Beischriften Compassio beate virginis Marie (Kl. 114) beziehungsweise Passio domini nostri Christi (Kl. 111) ${ }^{42}$ in den Handschriften setzen das vorgestellte Marienlied mit dem Passionslied in einen thematischen Bezug und weisen beide

40 Siehe den Hinweis im Editionsapparat in: Die Lieder Oswalds von Wolkenstein, S. 289: riel vermutlich bairisch ,Bündel, Wulst'.

41 Sowohl widerumb (II,3) als auch über (III,2) weisen durch die Aufeinanderfolge zweier unbetonter Silben jeweils einen Anapäst auf.

42 Vgl. ausführlich zum Passionslied Schnyder. 
Lieder als reziproke Stücke mit je unterschiedlichem Schwerpunkt aus: Während Kl. 111 die Passionsgeschichte in insgesamt elf Strophen detailliert darlegt und die Figur Marias sowie ihre compassio lediglich an wenigen Stellen erwähnt (I,16-18; VI,13-18; X,7-10; XI,5-6; XI,9-10), ${ }^{43}$ beschränkt sich Kl. 114 umgekehrt auf die wesentlichen, oftmals nur wenige Verse einnehmenden Passionsereignisse und setzt stattdessen den Fokus auf die Figur der mitleidenden Mutter. ${ }^{44}$ Die genannten Stationen des jeweils szenisch dargestellten Passionsweges Jesu sind mit Ausnahme der in beiden Stücken zitierten Speerprobe des Longinus biblisch gestützt, wenn auch die Passionsgeschichte im Vergleich zu den vier Evangelien hinsichtlich der Chronologie sowie der Vollständigkeit der Wegstationen geringfügig abweicht. ${ }^{45}$

Passions- und Marienlied stehen außerdem auch in intertextuellem Bezug, wie die folgenden Verse von Kl. 111 veranschaulichen: Maria, die vil raine magt, / unsäglich ward betrüebt, / als ir die mer ain junger sagt, / haiss wainen si da üebt / umb iren seligen lieben schatz, / den si empfieng, gepar durch keuschen latz (VI,13-18). Die genannten Verse, die analog zu Kl. 114 die Nachricht an Maria über die Verhaftung Jesu anführen, können geradezu als der semantische Anknüpfungspunkt des in den Handschriften jeweils einige Blätter später folgenden Marienliedes gelten, das ebendiese mer zum Anlass für die Narration der compassio Marias nimmt. Gestützt wird die Hypothese des engen thematischen Bezuges beider Stücke durch zusätzliche intertextuelle Verweise. Die scholastische Satisfaktionslehre, die in der zweiten Strophe des Marienliedes anklingt (II,8-11), ist im Passionslied noch deutlicher ausgestaltet: Auf das Glaubensmysterium des Todes Jesu in der dritten Strophe (III,8-12) folgt unter Berufung auf Thomas von

43 Das weitaus detailliertere Passionsgeschehen in Kl. 111 beginnt in Strophe IV und knüpft an die ,Vorgeschichte، in den Strophen I bis III (Sündenfall; Höllenfahrt Jesu und Befreiung der gerechten Seelen) an: Verrat und Judaskuss auf dem Ölberg; Gebet auf dem Ölberg und Rückkehr zu den schlafenden Jüngern (Strophe IV); Verrat durch Judas (Strophe V); Verhaftung Jesu auf dem Ölberg und dreimalige Verleugnung durch Petrus (Strophe VI); Verhör Jesu durch Pilatus und Herodes; Kleiderraub vor Pilatus (Strophe VII); Jesus wird unter Beschimpfung vor Pilatus gebracht; Geißelung und Dornenkrönung; Vorführung Jesu vor die wartenden Menschen, die Jesu Tod fordern (Strophe IX); Pilatus wäscht seine Hände in Unschuld; Kreuzauferlegung (Strophe X); Kreuzigung; Jesus überantwortet Maria seinem Jünger, Verzweiflung Jesu (Strophe XI samt Autorsignatur im letzten Vers: mir Wolkenstein werd dort sein huld berait [XI,18]). Die sogenannte Longinus-Strophe, welche die Speerprobe und Heilung des blinden Longinus thematisiert, folgt als Zusatz am Schluss des Liedes.

44 Vgl. zu den Stationen des Passionsgeschehens in Kl. 114 S. 111-112.

45 Der im Johannesevangelium genannte Soldat, der mit Hilfe einer Lanze in die Körperseite des toten Jesus am Kreuz sticht (Joh 19,34), wird im apokryphen Nikodemus-Evangelium als Longinus benannt; die Heilung des blinden Longinus, wie sie Kl. 111 in der an das Lied anschließenden Zusatzstrophe zitiert, entstammt wiederum der legendarischen Erzähltradition. 
Aquin die theologische Erklärung für den Gegensatz von übermäßigem Leiden des Gottessohnes und der Allmacht Gottvaters: Aquinas Thomas des beschaidt: / ain kindlin unversert / von lieb und durch gerechtikait / sich gab der marter swert, / wie wol sein vatter manigvalt / erlösen mocht den val durch sein gewalt (III,13-18). Passions- und Marienlied teilen mit der temporalen Angabe diesselbig nacht sogar eine wörtliche Referenz, die sowohl in der sechsten Strophe von Kl. 111 (VI,7) als auch in der zweiten Strophe von Kl. 114 (II,12) auf die Gefangennahme Jesu Bezug nimmt und in die Szene der Verleugnung durch Petrus mündet.

\section{Fazit}

Wie die Analyse von Kl. 114 verdeutlicht, steht der Figurenkörper, vornehmlich derjenige Marias, als Träger der Emotionen Schmerz und Trauer im Mittelpunkt. Bedenkt man den performativen Aspekt von Emotionalität - d.h. Emotionen werden nicht nur verbal und nonverbal inszeniert, sondern setzen Handlungen allererst in $\mathrm{Gang}^{46}$-, so weist das Lied hinsichtlich der intendierten compassio über die implizite Bedeutungsebene hinaus. Das Leid des Gottessohnes, das anhand des Blutes körperlich visualisiert wird, überträgt sich zunächst textintern auf die zusehende und zuhörende Gottesmutter. Während die passio Christi die compassio Mariae textintern auslöst, zielt vor allem das narrativierte Mitleiden der Gottesmutter textextern auf die compassio der Zuhörerschaft. Der Figurenkörper als Medium der Vermittlung von compassio stellt Oswalds Marienlied in den Kontext der spätmittelalterlichen Marienklagen beziehungsweise im weitesten Sinn der Passionsspiele ${ }^{47}$ - die genannten Texttypen sind mit dem vorgestellten Marienlied insofern wirkungsäquivalent, als dass sich inneres Erleben und äußerlich sichtbarer Schmerz (in Marienklage und Passionsspiel) beziehungsweise imaginierter Schmerz (in Kl. 114) textintern und textextern überlagern, um die compassio auf Figurenebene und bei der Zuhörerschaft gleichermaßen zu erwirken. Maria kommt hierbei eine Vermittlerfunktion zu, denn in ihrer eigenen compassio wird sie zur Identifikationsfigur, welche die Heilsgeschichte für das Publikum emotional beglaubigt. ${ }^{48}$ Ohne an dieser Stelle kausale Zusammenhänge, geschweige denn eine Gattungsüberschneidung von Oswalds Marienlyrik und spätmittelalterlichen Passionsspielen konstatieren zu wollen,

46 Vgl. Koch, S. 58.

47 Auch im Passionsspiel steht, wie Schulze: Schmerz, S. 230, verdeutlicht, der Figurenkörper im Mittelpunkt der compassio-Inszenierung - „[d]er leiblich sichtbare Schmerz korrespondiert mit dem gefühlhaft erlebten Leid.“

48 Vgl. Schulze: Planctus, S. 204. 
lassen sich vor dem Hintergrund der spätmittelalterlichen Passionsfrömmigkeit durchaus inhaltliche Analogien feststellen. So finden sich das Motiv der Ohnmacht Marias als Sinnbild für die Übermacht des Schmerzes sowie die Verwundung der Gottesmutter durch die Marterinstrumente der Passion auch in den Passionsspielen, beispielsweise im ,Donaueschinger Passionsspiel ' (letztes Drittel des fünfzehnten Jahrhunderts). ${ }^{49}$

Der Figurenkörper steht in Oswalds Marienlied auch im Fokus der Inszenierung der Blickrichtung der Rezipienten und Rezipientinnen. Während Maria zu Beginn über einen Dritten von dem Passionsgeschehen erfährt, in der zweiten Strophe die Abführung ihres Sohnes von Weitem hört und sieht, ist sie in der vierten Strophe bei der Kreuzauferlegung selbst anwesend und in der letzten Liedstrophe schließlich am Fuße des Kreuzes angelangt. Diese Bewegungsinszenierung auf Textebene, die Maria immer näher an das Passionsgeschehen beziehungsweise die Figur des leidenden Christus heranrückt, endet am Kreuz und entspricht dem textexternen inneren Blick der Rezipientinnen und Rezipienten im Zuge der Vergegenwärtigung des gehörten Passionsgeschehens. Die Aufmerksamkeit richtet sich auf den Ziel- und Endpunkt nicht nur des Liedes, sondern die christliche Heilsbotschaft - den Erlösertod Jesu.

Die Inszenierung von Bewegung und Blicken korrespondiert mit der überwiegend nonverbalen Codierung von compassio. Lediglich zweimal ist eine verbale Codierung von Emotion greifbar, und zwar - dem berichtenden Duktus des Liedes entsprechend - stets im Kontext des Text-Ichs selbst: so in der zweiten Strophe, wenn es Maria unzälich klag zugesteht, und in der letzten Strophe, als es die mangelnden Zeugnisse der ellend wainleich klag bezeugt; Maria selbst meldet sich nicht zu Wort. Im Unterschied zur Marienklage, die Maria zum Subjekt der Klage erhebt, bleibt die Gottesmutter in Kl.114 Objekt der Klage des Text-Ichs. Im Zentrum der Vermittlung von passio und compassio steht hier vor allem die nonverbale, körperliche Inszenierung von Emotion, die zum Stimulans des Affektes wird: Der Schmerz, der Jesus zweimal zu Boden wirft, erfasst auch Maria, die insgesamt dreimal in Ohnmacht fällt; die Folterinstrumente Jesu verwunden auch Maria körperlich. ${ }^{50}$ Die sich am Körper Marias manifestierenden Schmerzen zeichnen sie als Beteiligte am Erlösungsgeschehen und -werk aus, sie wird zur vorbildhaften mediatrix, die für die Menschen modellhaft die compassio vorführt und in ihrer Rolle als Mittlerin zwischen Immanenz und Transzendenz die Teil-

49 Vgl. Schulze: Schmerz, S. 223 u. 226.

50 Erinnert sei an dieser Stelle nochmals an den Geißelschlag, der Maria zu Boden wirft (III,6f.), sowie an den Speerstich (V,18f.), der zur Metapher des Leidensschwertes wird. 
habe am Heilsgeschehen vorprägt. ${ }^{51}$ Auch die Blickregie, die im Passionsspiel den gemarterten Körper Jesu, in der Marienklage die bis zum körperlichen Schmerz trauernde Mutter in den Fokus der Betrachtung stellt, findet sich, wie bereits erwähnt, in übertragener Art und Weise ebenfalls in Oswalds Marienlied: Ebenso wie Maria auf Figurenebene am Fuß des Kreuzes anlangt, gilt schließlich die Aufmerksamkeit der Rezipienten und Rezipientinnen - vermittelt über die durch die Gottesmutter vorgeprägte Emotion des Mitleidens - dem Gekreuzigten und dem Zielpunkt christlichen Heilsgeschehens.

Der affektive Kontaktbereich zwischen Immanenz und Transzendenz, den die compassio stimuliert und eröffnet, wird letztlich durch die im gesamten Lied immer wieder präsente jeweilige Doppelrolle Marias und Jesu konstituiert: Jesus wird neben seiner im Leid zur Schau gestellten Menschlichkeit ebenso als verherrlichter Gottessohn gepriesen; Maria wird neben ihrem menschlichen Schicksal als Mutter, die ihren Sohn verliert, gleichermaßen in ihrer Rolle als göttlich erwählte und jungfräuliche Mutter des Erlösers ${ }^{52}$ hervorgehoben (vgl. III,1: gots erwelte creatur). Auf diese Weise erscheint sie als eine über die Menschen bereits erhabene Figur, ohne freilich selbst göttlich zu sein. Der Kontrast von menschlichem Leid und göttlicher Erwählung bei Maria sowie die zweifache Wesenheit Jesu als leidender Mensch und glorifizierter Heiland intensiviert das im Lied dargestellte Leid noch zusätzlich, wenn beispielsweise der preisende Ehrentitel für Maria mit der Interjektion des Text-Ichs zusammengeführt wird: $O$ junckfröuliche raine maid, / mich wundert, das dir nit erbrach / dein keuschlich herz von grossem laid, / als du den herren hort und sach (II,8-11).

Die Figur Marias nimmt im Lied eine entscheidende Doppelfunktion ein: Zum einen ist sie im Passionsbericht des Text-Ichs einmalig Beteiligte am Passionsgeschehen; zum anderen erscheint sie als Vorbildfigur für die Gläubigen. Im imaginativen Mitschauen und Mitschreiten der Zuhörerschaft mit der im Mittelpunkt stehenden Figur Marias entfaltet die compassio im affektiven Mit- und Nachvollzug des Mitleidens der Gottesmutter ihr performatives Potential und wird

51 Vgl. Schulze: Schmerz, S. 219. Ob man mit Ursula Schulze (ebd., S. 221) Maria aufgrund ihrer Funktion, die compassio für die Menschen zu präformieren, zugleich die Rolle der Miterlöserin zusprechen kann, scheint mir fraglich und müsste anhand einzelner Passionsspiele und/oder Marienklagen noch weiter verifiziert werden. Zwar wird die Gottesmutter gerade in der spätmittelalterlichen Frömmigkeit nicht selten tatsächlich zur corredemptrix erhoben, die bloße compassio Mariae spricht Maria meiner Meinung nach jedoch noch keinen Subjektstatus im göttlichen Heilsplan zu - Maria bleibt als mediatrix göttlich erwähltes Objekt.

52 Maria wird mit Hilfe marianischer Ehrentitel mehrmals als jungfräuliche und göttlich erwählte Mutter Jesu gepriesen: I,2; I,6f.; II,8-11; III,1; III,15; III,18. Christus als Gottessohn und Erlöser ist in den folgenden Abschnitten Gegenstand des Preises: I,3-5; IV,2. 
heilswirksam. ${ }^{53}$ Im Vollzug des Marienliedes wird die soteriologische Wirkung der compassio immer wieder neu memoriert und aktualisiert. Ähnlich wie in der Marienklage oder dem Passionsspiel steht die „affektive Gotteserkenntnis“54 im Zentrum von Kl. 114, welche die emotionale Vergegenwärtigung des christlichen Heilsgeschehens über die mentale Einsicht in das Theologumenon der Leidensbereitschaft Gottes stellt..$^{55}$

\section{Literaturverzeichnis}

Beinert, Wolfgang: Die mariologischen Dogmen und ihre Entfaltung. In: Handbuch der Marienkunde. Hg. von dems., Heinrich Petri. Regensburg 1984, S. 232-314.

Berger, Lothar: Die ,Goldene Muskate‘. Ein spätmittelalterlicher Passionstraktat. Edition und Untersuchungen. Marburg 1969.

Die Lieder Oswalds von Wolkenstein. Hg. von Karl Kurt Klein. 4., grundlegend neu bearb. Aufl. von Burghart Wachinger. Berlin u. a. 2015 (Altdeutsche Textbibliothek 55).

Egidi, Margreth: Theatralität und Bild im spätmittelalterlichen Passionsspiel. Zum Verhältnis von Gewaltdarstellung und compassio. In: Räume der Passion. Raumvisionen, Erinnerungsorte und Topographien des Leidens Christi in Mittelalter und Früher Neuzeit. Hg. von Hans H. Aurenhammer, Daniela Bohde. Bern u. a. 2015 (Vestigia bibliae 32/33), S. $181-203$.

Eming, Jutta u. a.: Emotionalität und Performativität in narrativen Texten des Mittelalters. In: Paragrana 10 (2001), S. 215-233.

Hartmann, Sieglinde: Gotische Madonnenbilder und die Marienlyrik Oswalds von Wolkenstein. In: wort und wîse, singen unde sagen. Fs. für Ulrich Müller. Hg. von Ingrid Bennewitz. Göppingen 2007 (GAG 741), S. 71-92.

Hartmann, Sieglinde: Oswald von Wolkenstein heute. Traditionen und Innovationen. In: Jahrbuch der Oswald von Wolkenstein Gesellschaft 15 (2005), S. 349-372.

Koch, Elke: Trauer und Identität. Inszenierungen von Emotionen in der deutschen Literatur des Mittelalters. Berlin u. a. 2006 (Trends in Medieval Philology 8).

Kraß, Andreas: Stabat mater dolorosa. Lateinische Überlieferung und volkssprachliche Übertragungen im deutschen Mittelalter. München 1998.

Langer, Otto: Passio und Compassio. Zur Bedeutung der Passionsmystik bei Bernhard von Clairvaux. In: Die dunkle Nacht der Sinne. Leiderfahrung und christliche Mystik. Hg. von Gotthard Fuchs. Düsseldorf 1989, S. 41-62.

Lentes, Thomas: Inneres Auge, äußerer Blick und heilige Schau. Ein Diskussionsbeitrag zur visuellen Praxis in Frömmigkeit und Moraldidaxe des späten Mittelalters. In: Frömmigkeit

53 Wie Stefan Rosmer festhält, kann das besagte Marienlied nicht zuletzt aufgrund des Anspruchs, verbindliche Aussagen über theologische Kernpunkte zu treffen und objektives Heilswissen zu vermitteln, in die Tradition der Sangspruch- und Meisterlieddichtung gestellt werden. Vgl. Rosmer, S. 265 u. 267.

54 Schulze: Schmerz, S. 212.

55 Vgl. ebd. 
im Mittelalter. Politisch-soziale Kontexte, visuelle Praxis, körperliche Ausdrucksformen. Hg. von Klaus Schreiner. München 2002, S. 179-220.

Löser, Freimut: Oswalds von Wolkenstein geistliche Dichtung. In: Oswald von Wolkenstein im Kontext der Liedkunst seiner Zeit. Hg. von Ingrid Bennewitz, Horst Brunner. Wiesbaden 2013 (Jahrbuch der Oswald von Wolkenstein Gesellschaft 19 [2012/2013]), S. 7- 30.

Petersen, Nils Holger: Devotion and Dramaticity in the Bordesholmer Marienklage (1476). In: Dies est leticie. Essays on Chant in Honour of Janka Szendrei. Hg. von David Hiley, Gábor Kiss. Ottawa, Kanada 2008 (Wissenschaftliche Abhandlungen XC; Musicological Studies XC), S. $413-427$.

Rosmer, Stefan: Zusammenhänge zwischen metrisch-melodischen Formtypen, sprachlichen Gestaltungsmustern und Inhalten in den Marienliedern Oswalds. In: Oswald von Wolkenstein im Kontext der Liedkunst seiner Zeit. Hg. von Ingrid Bennewitz, Horst Brunner. Wiesbaden 2013 (Jahrbuch der Oswald von Wolkenstein Gesellschaft 19 [2012/2013]), S. 255-272.

Ruh, Kurt: Zur Theologie des mittelalterlichen Passionstraktats. In: Theologische Zeitschrift 6 (1950), S. 17-39.

Satzinger, Georg, Hans-Joachim Ziegeler: Marienklagen und Pietà. In: Die Passion Christi in Literatur und Kunst des Spätmittelalters. Hg. von Walter Haug, Burghart Wachinger. Tübingen 1993 (Fortuna vitrea 12), S. 241-276.

Schulze, Ursula: Planctus ante nescia. Mutter und Sohn: Maria und Jesus im Geistlichen Spiel des späten Mittelalters. In: Generationenkonflikte auf der Bühne. Perspektiven im antiken und mittelalterlichen Drama. Tübingen 2007 (Drama. Studien zum antiken Drama und zu seiner Rezeption. Neue Serie 3), S. 197-210.

Schulze, Ursula: Schmerz und Heiligkeit. Zur Performanz von Passio und Compassio in ausgewählten Passionsspieltexten (Mittelrheinisches, Frankfurter, Donaueschinger Spiel). In: Forschungen zur deutschen Literatur des Spätmittelalters. Fs. für Johannes Janota. Hg. von Horst Brunner, Werner Williams-Krapp. Tübingen 2003, S. 211-232.

Schnyder, André: Das erste Passionslied Oswalds von Wolkenstein. Eine Interpretation von Kl. 111, mit einem Seitenblick auf G 23 des Mönchs von Salzburg. In: Speculum Medii Aevi 2 (1996), S. 43-58.

Spechtler, Franz Viktor: Beiträge zum deutschen geistlichen Lied des Mittelalters III: Liedtraditionen in den Marienliedern Oswalds von Wolkenstein. In: Gesammelte Vorträge der 600-Jahrfeier Oswalds von Wolkenstein. Seis am Schlern 1977. Dem Edlen unserm sunderlieben getrewen Hern Oswaltten von Wolckenstain. Hg. von Hans-Dieter Mück, Ulrich Müller. Göppingen 1978 (GAG 206), S. 179-203. 